\title{
Spin Physics in Deep-Inelastic Semi-Inclusive Reactions with an 11-GeV Electron Beam at Hall A of Jefferson Laboratory
}

\author{
Xiaodong Jiang \\ Department of Physics and Astronomy, Rutgers University, Piscataway, New Jersey.
}

\begin{abstract}
We outline the physics oppertunities of semi-inclusive deep inelastic measurements with a polarized $\mathrm{NH}_{3}$ and a polarized ${ }^{3} \mathrm{He}$ target in Jefferson Lab Hall A after the planned $12 \mathrm{GeV}$ CEBAF machine upgrade. In this paper, we estimate statistical uncertainties associated with doublespin and single-spin asymmetries in $\left(e, e^{\prime} \pi\right)$ type measurements.
\end{abstract}

\section{INTRODUCTION}

With the planned $12 \mathrm{GeV}$ upgrade at the Thomas Jefferson National Accelerator Facility in Newport News, Virginia, the combination of a high current CW polarized electron beam and the use of high density polarized targets presents many new physics opportunities, especially in the measurements of spin observables of deep inelastic semi-inclusive scattering (SIDIS) reactions. If factorization between quark scattering and quark fragmentation can be clearly demonstrated, SIDIS can provide direct accesses to quark and quark polarization distributions. The unique feature of quark-flavor tagging capability allows us to study the flavor decomposition of the nucleon spin structure and to access new distribution functions such as the quark transversity distributions.

At the experimental Hall A, after the $12 \mathrm{GeV}$ CEBAF machine upgrade, $85 \%$ polarized electron beam with a current of $40 \mu \mathrm{A}$ can be delivered up to a beam energy of 11 $\mathrm{GeV}$. Luminosities of $10^{38} \mathrm{~cm}^{-2} \mathrm{~s}^{-1}$ can be achieved for unpolarized hydrogen or deuterium targets, a factor of $2 \times 10^{5}$ improvement over the typical HERMES luminosity of unpolarized targets. Assuming no improvement on the polarized target technology, a polarized proton luminosity of $10^{35} \mathrm{~cm}^{-2} \mathrm{~s}^{-1}$ with $80 \%$ polarization can be achieved on a polarized $\mathrm{NH}_{3}$ target, and a ${ }^{3} \mathrm{He}$ luminosity of $10^{36} \mathrm{~cm}^{-2} \mathrm{~s}^{-1}$ with $45 \%$ polarization can be achieved with a polarized ${ }^{3} \mathrm{He}$ gas target, an improvement of four orders of magnitudes over the HERMES luminosity in each case.

A new moderate resolution $\left(\delta p / p=10^{-3}\right)$ magnetic spectrometer, the Medium Acceptance Device (MAD), is under consideration for Hall A. The MAD spectrometer will operate up to $6 \mathrm{GeV} / \mathrm{c}$ in central momentum with a momentum acceptance of $\pm 15 \%$. The geometrical acceptance of MAD will be ranging from $6 \mathrm{msr}$ at $13^{\circ}$ to $30 \mathrm{msr}$ at $35^{\circ}$. In a typical SIDIS measurement, the MAD spectrometer can be used as the electron arm with the the existing Hall A HRS spectrometer serves as the hadron arm. The HRS spectrometer, operates up to $4.3 \mathrm{GeV} / \mathrm{c}$ in central momentum, has a momentum acceptance of $\pm 4.5 \%$ and a solid angle of 6 msr. With an additional SEPTUM magnet, the HRS 
spectrometer is able to access $6^{\circ}$ in scattering angle.

\section{AVAILABLE KINEMATICS}

At a beam energy of $11 \mathrm{GeV}$, the accessible kinematics region in $\left(x, Q^{2}\right)$ and $\left(x, W^{2}\right)$ plane are shown in Fig. 1. Constant electron scattering angle $\left(\theta_{e}\right)$ lines are plotted from $10^{\circ}$ to $60^{\circ}$ in addition to the constant $\vec{q}$ angle $\left(\theta_{q}\right)$ lines. In order to stay in the deep inelastic region at the highest possible $\mathrm{W}$, the center of the fragmentation cone is limited to a forward angle of $\theta_{q}>15^{\circ}$.

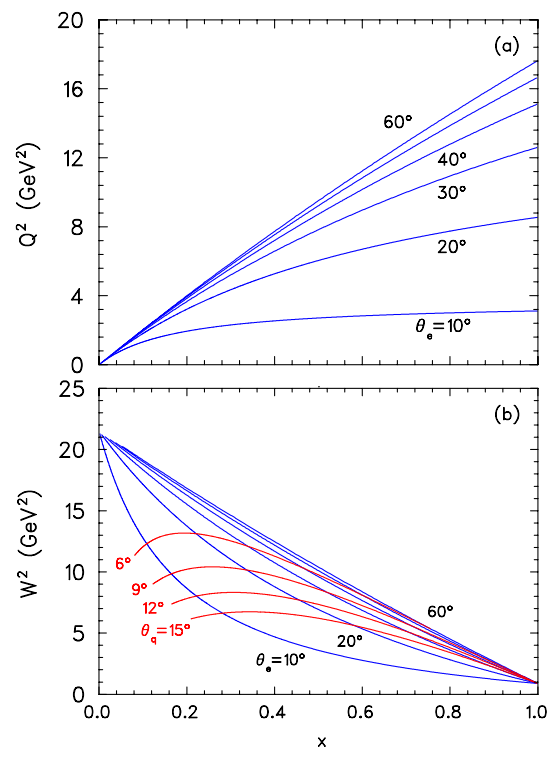

FIGURE 1. Accessible kinematics region for an $\left(e, e^{\prime} h\right)$ measurement at $\mathrm{E}_{0}=11 \mathrm{GeV}$.

In a double-spin asymmetry measurement, the fragmented hadron will be detected along the momentum transfer direction $\vec{q}$, and the target spin will be aligned along the same direction, as illustrated in Fig. 2(left). In a single-spin asymmetry measurement, the fragmented hadron will be detected on the side of $\vec{q}$, within the electron scattering plane as illustrated in Fig. 2(right). The target spin will be aligned in a plane perpendicular to $\vec{q}$, and the Collins angle $\phi$ is defined as the angle between the target spin and the $\left(\vec{q}, \vec{p}_{\pi}\right)$ plane.

\section{DOUBLE SPIN ASYMMETRIES IN SIDIS}

While unpolarized semi-inclusive meson production provides means of extracting spinaveraged quark and antiquark distributions in the nucleon, semi-inclusive production with a polarized beam on a polarized target offers the prospect of determining the spindependence of the individual quark species. Furthermore, by comparing semi-inclusive 


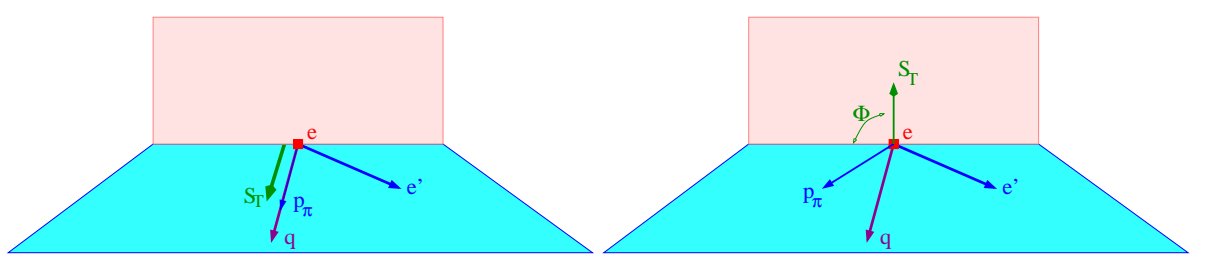

FIGURE 2. Target spin configuration in a double-spin asymmetry measurement (left), and in a singlespin asymmetry measurement (right), viewed toward the electron beam.

data with inclusive DIS measurements, one can directly test the degree to which flavor SU(3) symmetry holds in DIS processes. At large $Q^{2}$, the spin asymmetry $A_{1}^{h}$ for the production of a hadron $h$ by a polarized virtual photon on a polarized nucleon can be written:

$$
A_{1}^{h}(x, z)=P_{e} \cdot P_{T} \cdot \frac{y\left(1-\frac{1}{2} y\right)}{1-y+\frac{1}{2} y^{2}} \cdot \frac{\sum_{q} e_{q}^{2} \Delta q(x) D_{q}^{h}(z)}{\sum_{q} e_{q}^{2} q(x) D_{q}^{h}(z)}
$$

where $P_{e}$ and $P_{T}$ are beam and target polarization. Measurement of $\pi^{+}$and $\pi^{-}$(or $K^{+}$and $K^{-}$) mesons from proton or neutron targets, together with knowledge of the unpolarized distributions $q(x)$, allows one to extract from Eq. (1) information on the spin-dependent distributions $\Delta q(x)$ and $\Delta \bar{q}(x)$.

Assuming the use of a polarized ${ }^{15} \mathrm{NH}_{3}$ target and a polarized ${ }^{3} \mathrm{He}$ target in their standard configurations, a total of 1000 hour measurements on each target at $z=E_{\pi} / v=$ $0.40 \sim 0.5$ in each setting will yield high statistical accuracies on $A_{1}^{\pi^{+}}$and $A_{1}^{\pi^{-}}$, as shown in Fig. 3. The measurement time is arranged such that similar statistical accuracies can be achieved for $A_{1}^{\pi^{+}}$and $A_{1}^{\pi^{-}}$. Assuming factorization has been clearly demonstrated, the "purity" method used by the HERMES collaboration can be adopted to extract the quark polarization distributions from the measured semi-inclusive asymmetries [1]. The corresponding statistical accuracies are shown in Fig. 4 together with the HERMES published results [1] for comparison.

\section{SINGLE-SPIN ASYMMETRIES IN SIDIS}

Following Ref. [2], we calculated single-spin asymmetry $A_{T}$ as a function of $x, y$ and $z$ for $11 \mathrm{GeV}$ electron beam energy. Typical results of a 1000 hour measurement are shown in Fig. 5, where the $z$-dependence of $A_{T}$ are plotted for $x=0.2, Q^{2}=2.5 \mathrm{GeV}^{2}$ and $x=0.3, Q^{2}=3.0 \mathrm{GeV}^{2}$ kinematics. We assume $\delta q(x)=\Delta q(x)$ in this calculation. The AAC parameterization of the polarized nucleon structure functions were used for $\Delta q(x)$, and the CTEQ5M parameterization were used for the unpolarized structure functions. For the fragmentation functions, the parameterization of Aubert et al. [3] was adopted. We assume a typical polarized ${ }^{15} \mathrm{NH}_{3}$ target with $80 \%$ polarization, and a ${ }^{3} \mathrm{He}$ target of $45 \%$ polarization. The dilution factors due to the unpolarized nucleons in the target material have been taken into account. It is interesting to note that the $\pi^{-}$production on 


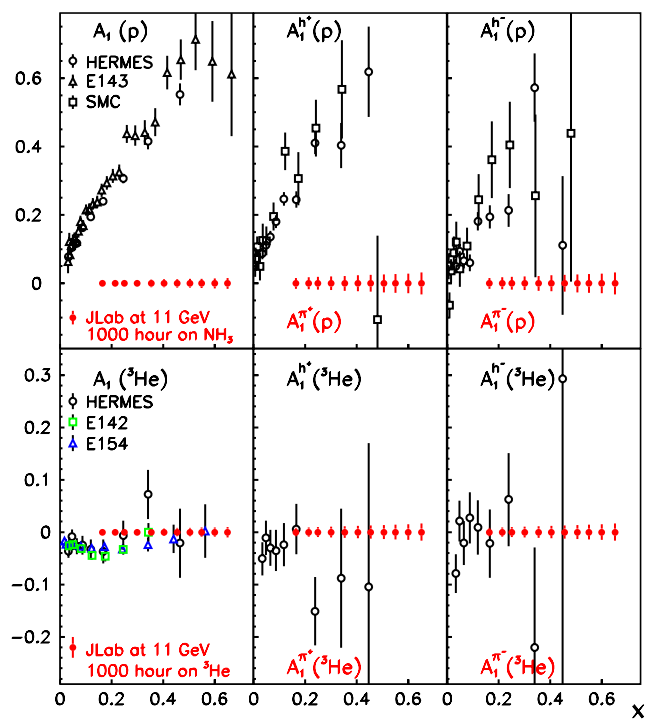

FIGURE 3. Expected semi-inclusive asymmetry measurements with polarized $\mathrm{NH}_{3}$ and ${ }^{3} \mathrm{He}$ targets. 1000 hours of beam time is assumed for each target. Error bars are statistical only.

a polarized ${ }^{3} \mathrm{He}$ target has a much larger asymmetry with an opposite sign compare to that of the $\pi^{+}$asymmetry. This trend is very different from the situation of a polarized proton target due to the contribution of d-quark transversity $\delta d(x)$ in the neutron.

\section{ACKNOWLEDGMENTS}

This work represents a collective effort by the Jefferson Lab Hall A collaboration. The author thanks H. Avagyan, T. Averett, J.-P. Chen, H. Gao, R. Gilman, D. Higinbotham, X. Ji, J. LeRose, W. Melnitchouk, Z.-E. Meziani, J.-C. Peng, R. Ransome for many discussions. This work is supported by the US Department of Energy and the National Science Foundation.

\section{REFERENCES}

1. K. Ackerstaff et al., Phys. Lett. B 464, 123 (1999).

2. V.A. Korotkov, W.-D. Nowak and K.A. Oganessian, Eur. Phys. J. C 18, 639 (2001).

3. J.J. Aubert et al., Phys. Lett. B 160, 417 (1985). 


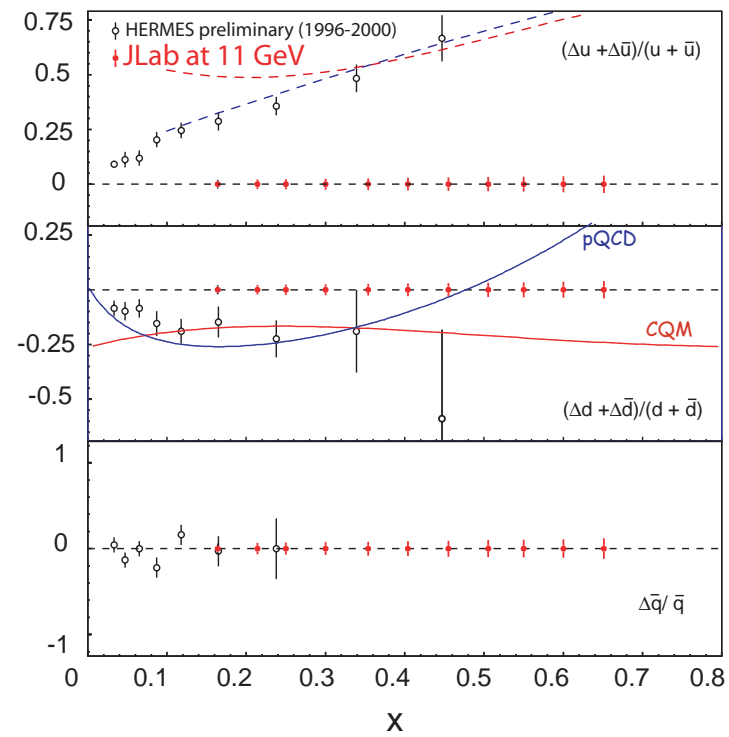

FIGURE 4. Expected results on $\Delta \bar{q} / \bar{q}$ from semi-inclusive asymmetry measurements with polarized $\mathrm{NH}_{3}$ and ${ }^{3} \mathrm{He}$ targets. 1000 hours of beam time is assumed for each target. Error bars are statistical only.

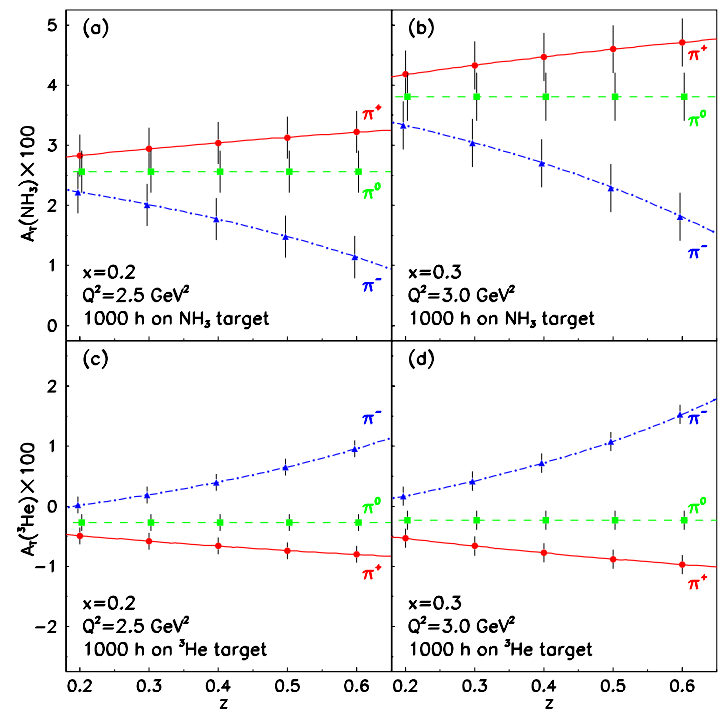

FIGURE 5. The expected precisions in transverse single-spin asymmetry measurements on a polarized ${ }^{15} \mathrm{NH}_{3}$ and a polarized ${ }^{3} \mathrm{He}$ target, for the kinematics of $x=0.2$ and $x=0.3 .1000$ hours of beam time is assumed for each target. 